\title{
Stadienabhängige Routine-Checks beim Augenarzt empfohlen
}

Fragestellung: In welchen Abständen muss man Typ-1-Diabetikern fachärztlich kontrollieren, um behandelbare Retinopathien rechtzeitig zu erkennen?

Hintergrund: Die diabetische Retinopathie ist die häufigste Ursache für Erblindung. Wenn sich ein Maculaödem oder eine bedrohliche proliferative Retinopathie entwickelt, können spezifische Maßnahmen wie Laserkoagulation, intraokuläre Glukokortikoide oder Antikörper gegen vaskuläre endotheliale Wachstumsfaktoren (anti-VEGF) dem drohenden Visusverlust entgegenwirken. Es kommt deshalb darauf an, diese Veränderungen rechtzeitig und zuverlässig zu erkennen.

Patienten und Methoden: Im ursprünglichen Diabetes Control and Complications Trial (DCCT) wurden 1.441 Typ-1-Diabetiker im Alter zwischen 13 und 39 Jahren ohne oder mit nur leichter Retinopathie aufgenommen. Von 1983 bis 1993 erhielten die Patienten nach Zufallskriterien entweder eine intensive oder eine konventionelle antidiabetische Therapie, seit 1994 dann alle die gleiche intensive Therapie. Seit nun über 30 Jahren wurden in Abständen
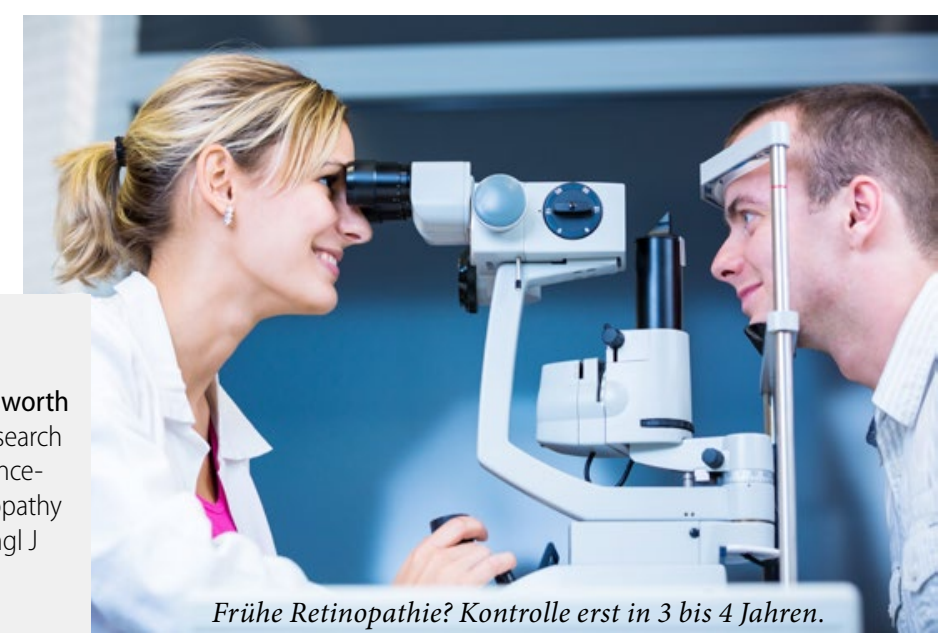

T Empfohlener Abstand von Verlaufsuntersuchungen in Abhängigkeit vom Retinopathie-Stadium

\begin{tabular}{lllll}
\hline & Stadium 1 & Stadium 2 & Stadium 3 & Stadium 4 \\
Screening & 4 Jahre & 3 Jahre & 6 Monate & 3 Monate \\
\hline
\end{tabular}

von 6 bis 48 Monaten insgesamt 23.961 Fundoskopien fotografisch dokumentiert und die üblichen Risikofaktoren für eine Retinopathie erfasst. Aus dieser Datenbasis werden rationale Empfehlungen für das Retinopathie-Screening abgeleitet.

Ergebnisse: Bei Unterscheidung von 5 Retinopathiegraden (keine, milde, moderate und schwere non-proliferative sowie proliferative oder Makulaödem) zeigte sich: Mit dem Schweregrad verkürzt sich die Zeit bis zur Progression ganz erheblich. Nur 2,9\% der Patienten im Stadium 1 erreichen das Stadium 5 innerhalb von 4 Jahren, aber 3,7 \%, 6,6 \% und 14,4\% derjenigen in den Stadien 2 bis 4 in nur 36, 6 bzw. 3 Monaten. Wenn man anstrebt, die Progression zu Stadium 5 und damit den Zeitpunkt für eine spezifische Therapie mit einer Wahrscheinlichkeit von $95 \%$ zu erfassen, errechnen sich die in der $>$ Tab. 1 genannten Abstände für Fundoskopien. Das mittlere $\mathrm{HbA}_{1 \mathrm{c}}$, nicht aber andere Risikofaktoren beeinflussen die Abstände der Verlaufsuntersuchungen.

Schlussfolgerung: Die errechneten individualisierten ScreeningAbstände führen zu einer zuverlässigeren Diagnose der Retinopathie bei weniger Fundoskopien.

\section{- Kommentar von Prof. Dr. med. Heinrich Holzgreve}

\section{Evidenzbasierte maßgeschneiderte Augenkontrollen}

Bislang wurde aufgrund überholter epidemiologischer Daten ein Beginn des Retinopathie-Screenings 3 - 5 Jahre nach Diagnose des Typ-1-Diabetes empfohlen. Die Progression der Retinopathie ist aber ganz entscheidend vom Ausgangsbefund abhängig. Deshalb können Verlaufsuntersuchungen bei fehlenden oder geringen Ausgangsbefunden in größeren Abständen von Jahren erfolgen, sind aber bei fortgeschrittener Schädigung immer häufiger, schließlich in Monatsabständen nötig. Derartige, am Patientenbefund orientierte Termine gewährleisten eine zeitgerechte Diagnose bei geringerem Aufwand.

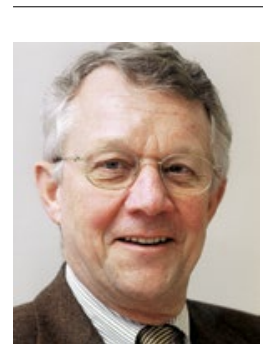

Prof. Dr. med. Heinrich Holzgreve

Internist, Kardiologische Praxis

Burgstr. 7, 80331 München

heinrich.holzgreve@t-online.de 